\title{
High Rab27A expression indicates favorable prognosis in CRC
}

\author{
Chuanbing Shi ${ }^{\dagger \dagger}$, Xiaojun Yang ${ }^{2 \dagger}$, Yijiang $\mathrm{Ni}^{3}$, Ning Hou${ }^{4}$, Li Xu ${ }^{4}$, Feng Zhan ${ }^{5}$, Huijun Zhu ${ }^{6}$, Lin Xiong ${ }^{7,8}$ \\ and Pingsheng Chen ${ }^{1 *}$
}

\begin{abstract}
Background: Rab27A is a peculiar member in Rab family and has been suggested to play essential roles in the development of human cancers. However, the association between Rab27A expression and clinicopathological characteristics of colorectal cancer (CRC) has not been elucidated yet.

Methods: One-step quantitative real-time polymerase chain reaction ( $q P C R$ ) test with 18 fresh-frozen CRC samples and immunohistochemistry $(\mathrm{IHC})$ analysis in 112 CRC cases were executed to evaluate the relationship between Rab27A expression and the clinicopathological features of CRC. Cox regression and Kaplan-Meier survival analyses were performed to identify the prognostic factors for 112 CRC patients.

Results: The results specified that the expression levels of Rab27A mRNA and protein were significantly higher in CRC tissues than that in matched non-cancerous tissues, in both qPCR test $(p=0.029)$ and IHC analysis $(p=0.020)$. The IHC data indicated that the Rab27A protein expression in CRC was statistically correlated with lymph node metastasis $(p=0.022)$ and TNM stage $(p=0.026)$. Cox multi-factor analysis and Kaplan-Meier method suggested Rab27A protein expression $(p=0.012)$ and tumor differentiation $(p=0.004)$ were significantly associated with the overall survival of CRC patients.
\end{abstract}

Conclusion: The data indicated the differentiate expression of Rab27A in CRC tissues and matched non-cancerous tissues. Rab27A may be used as a valuable prognostic biomarker for CRC patients.

Keywords: Rab27A, CRC, qPCR, IHC, Metastasis, Prognosis

\section{Background}

Colorectal cancer (CRC) is the third most common cancer and more than 1 million people develop CRC annually [1]. It is reported that there are estimated 140,000 newly diagnosed cases of CRC and approximately 50,000 cancer deaths from CRC in America in 2013 [2, 3]. Of patients with primary CRC, nearly $20 \%$ encounter distant metastasis at the time of diagnosis and only $10-30 \%$ of patients with distant metastasis can have potentially curative resection of the primary tumor and the distant focus [4-6]. For CRC prognosis, survival is critically related to stage at diagnosis, with five-year survival rates of $90 \%$ for localized cases, while $67 \%$ for regional cases and only $10 \%$ for distant metastatic cases [7]. Although the great

\footnotetext{
* Correspondence: chenps@seu.edu.cn

${ }^{\dagger}$ Equal contributors

'Department of Pathology and Pathophysiology, School of Medicine, Southeast University, Nanjing 210009, China

Full list of author information is available at the end of the article
}

developments for diagnosis and treatment for CRC, the overall survival rate of CRC patients rarely shows the encouraging result and post-operative recurrence and metastasis remain the two most challenging obstacles [8]. Hence it is valuable and necessary to identify molecular predictive markers for the prognosis, which would facilitate the selection of therapeutic strategies and further ameliorate patients' survival for CRC [9].

The Rab family is a group of Ras-like small GTPases which locates in specific subcellular organelle and plays an important role in cell secretion, endocytosis, signal transduction, and development [10-12]. Rab27A is a peculiar member in Rab family for its specific role in human hereditary disease and dysfunction, such as Griscelli syndrome [13, 14]. Recently, several studies reported the diverse function of Rab27A in many kinds of human cancers. Differential expression of Rab27A was detected in murine xenografts of breast cancer (BC) metastasis

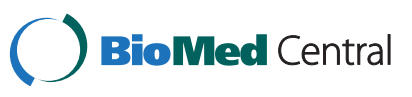


and Rab27A was highly associated with the invasive and metastatic potential of human BC cells [15]. Rab27A enhanced various ability of glioma cell, such as developing proliferation, promoting invasion and inhibiting apoptosis [16]. Rab27A also affected exosome secretion, modified tumor microenvironment and finally promoted tumor progression [17]. Moreover, two studies reported the prognostic role of Rab27A that high Rab27A expression indicated poor survival of patients with gliomas and hepatocellular carcinoma (HCC) $[18,19]$. All the above information suggest the significant relationship between Rab27A and cancer while the performance of Rab27A in $\mathrm{CRC}$ has yet to be explored. What function does Rab27A play in CRC deveolopment and what is the relationship between Rab27A expression and clinical features of CRC? We conducted this present research.

In this retrospective study, the mRNA and protein expression of Rab27A in CRC was investigated by onestep quantitative real-time polymerase chain reaction (qPCR) test and immunohistochemistry (IHC) analysis respectively. Subsequently, the correlations of Rab27A expression with the clinicopathological parameters of $\mathrm{CRC}$ were further explored. Finally, the survival analysis for identifying prognostic factors was executed.

\section{Methods}

\section{Ethics statement}

The Ethics Committee of the Affiliated Hospital of Nantong University and each local hospital approved the study protocol. A written informed consent and related pictures were also acquired from each patient for publication of this present research.

\section{CRC patient specimens}

Formalin-fixed, paraffin-embedded tumor samples from 112 CRC cases and 113 matched non-cancerous tissue specimens were collected in CRC patients from the Department of Pathology, the Affiliated Hospital of Nantong University from 2003 to 2008. Representative and important clinical information were collected from hospital medical records, including gender, age, tumor size, tumor location, histological type, tumor differentiation, serum carcino-embryonic antigen (CEA) level, primary tumor $(\mathrm{T})$, lymph node metastasis $(\mathrm{N})$, distant metastasis $(\mathrm{M})$, as well as TNM stage which was classified using the 7th American Joint Committee on Cancer (AJCC) staging system for CRC and overall survival status [20]. None of the patients received radiotherapy, chemotherapy, or immunotherapy prior to enterectomy.

\section{One-step qPCR analysis}

18 fresh CRC cancer tissue samples and 18 normal tumor-adjacent tissue samples were collected from the Department of Pathology, Affiliated Cancer Hospital of
Nanjing Medical University for qPCR analysis. Total RNA extraction, quality control, and one-step qPCR analysis were performed as previously described [21, 22].

The primers for Rab27A were as follows: forward primer 5'- GTA AGT GAC ATA GTA GTT -3' and reverse primer 5'- TTA TTC GTA GGT CTA ATG -3'. The glyceraldehyde 3-phosphate dehydrogenase (GAPDH) mRNA level was employed to standardize the measurements of the target gene and the primers for GAPDH were as follows: forward primer 5'-TGC ACC ACC AAC TGC TTA GC-3' and reverse primer 3'-GGC ATG GAC TGT GGT CAT GAG-5'. Amplification conditions consisted of $30 \mathrm{~min}$ at $42{ }^{\circ} \mathrm{C}$ for reverse transcription and 2 min at $94{ }^{\circ} \mathrm{C}$ for Taq activation, followed by 35 cycles at $95{ }^{\circ} \mathrm{C}$ for $20 \mathrm{~s}, 56{ }^{\circ} \mathrm{C}$ for $20 \mathrm{~s}$, and elongation at $72{ }^{\circ} \mathrm{C}$ for $30 \mathrm{~s}$. Each measurement was performed in triplicate.

\section{Tissue microarrays (TMA) construction and IHC analysis}

112 CRC and 113 normal tumor-adjacent tissues were enrolled in this present study. TMA was produced by the Department of Pathology, the Affiliated Hospital of Nantong University. Core tissue biopsies $(2 \mathrm{~mm}$ in diameter) were taken from individual paraffin-embedded sections and arranged in the new recipient paraffin blocks. TMA was cut into 4- $\mu \mathrm{m}$ sections and placed on super frost charged glass microscope slides. IHC analysis was performed as previously described [23-25]. Deparaffinized sections $(4 \mu \mathrm{m}$ thick) from array blocks were separately stained on an Autostainer Universal Staining System (LabVision, Kalamazoo, MI, USA) using mouse anti-Rab27A antibody (1:100, Abcam, Cambridge, UK). The secondary antibody used was horseradish peroxidaseconjugated antibody (Dako Cytomation, Carpinteria, CA, USA).

Rab27A immunostaining observation and evaluation were simultaneously performed by two independent pathologists. IHC results were analyzed according to a previously described method [24, 26, 27]. Staining intensity was scored as follows: 0 (negative), 1 (weakly positive), 2 (moderately positive), and 3 (strongly positive). The percentage of Rab27A-positive cells was also classified into 4 categories, where 1 was given for $0-10 \%, 2$ for $11-50 \%, 3$ for $51-80 \%$, and 4 for $81-100 \%$. The product of the intensity and percentage scores gave rise to the final Rab27A staining score. The degree of Rab27A staining was sorted by a two-level grading system, and staining scores were described as follows: a score below 4 $(<4)$ suggested low or no expression of Rab27A, while a score above 4 ( $\geq 4)$ suggested high expression of Rab27A.

\section{Statistical analysis}

The Rab27A mRNA expression normalized to GAPDH in CRC samples compared with matching non-cancerous tissue samples was analyzed with the Wilcoxon non- 
parametric signed-rank test. The relationships between Rab27A expression and clinicopathologic itemss of CRC were evaluated with $\chi^{2}$ tests. Survival curves were performed with Kaplan-Meier method. Univariate and multivariate Cox regression model were performed to detect the prognostic elements. For all tests, the significance level for statistical analysis was set at $p<0.05$. All data were analyzed using STATA Version 12.0 ((Stata Corporation, College Station, TX, USA).

\section{Results}

\section{Summarization of clinical data of 112 CRC patients}

A total of 112 CRC cases were collected from 73 men and 39 women and the representative clinical data are summarized in Table 1 . The mean age of all cases at the time of surgery was 65.14 years. There were 47 cases with tumor diameter $\geq 5 \mathrm{~cm}, 62$ with tumor diameter $<5 \mathrm{~cm}$. The tumor locations of 57 patients were in colon, 51 patients in rectum and 4 patients in ileocecal junction. The histological type of tumor in 102 cases was adenocarcinoma, while the other 10 cases were identified as non-adenocarcinoma type. 1 patient encountered a well-differentiated tumor, 92 suffered moderate tumor differentiation, and 15 had poor tumor differentiation. High serum CEA level $(\geq 15 \mathrm{ng} / \mathrm{ml})$ was observed in 14 cases, whereas low serum CEA level $(<15 \mathrm{ng} / \mathrm{ml})$ was displayed in 71 cases. The number of patients with T1, T2, T3 were 7, 26 and 79 respectively. Positive lymph node metastasis was observed in 44 patients while distant metastasis was noticed in 5 patients. According to TNM staging system, 29 patients were in stages I, 36 patients were in stages II, 42 patients were in stages III, whereas the remaining 5 patients were in stages IV.

\section{Detection of Rab27A mRNA expression by qPCR test}

Total RNA was extracted from 18 CRC tissues as well as matched tumor adjacent tissues, and then subjected to one-step qPCR to detect Rab27A mRNA expression. When normalizing to GAPDH, the means of Rab27A mRNA in CRC tissues and that of the corresponding non-cancerous tissues were calculated as $3.32 \pm 0.518$ and $1.76 \pm 0.444$, respectively $(\mathrm{t}=2.281, p=0.029)$. Rab27A expression in the CRC samples was nearly 1.89 -fold higher than that in matched non-cancerous tissues (Fig. 1).

\section{Detection of Rab27A protein expression by IHC}

High Rab27A expression was observed in 64 (57.1\%) of the 112 CRC samples compared with 47 (41.6\%) of 113 matched normal tumor-adjacent tissue samples. The difference was of statistically significance $\left(\chi^{2}=5.44, p=0.020\right)$ according to the $\chi^{2}$ test analysis and the data was agreeable with the previous qPCR test in which high level of Rab27A mRNA expression
Table 1 Correlation of Rab27A expression with clinicopathological characteristics of 112 CRC patients

\begin{tabular}{|c|c|c|c|c|c|}
\hline \multirow[t]{2}{*}{ Groups } & \multirow[t]{2}{*}{ No. } & \multicolumn{2}{|c|}{ Rab27A } & \multirow[t]{2}{*}{$x^{2}$} & \multirow{2}{*}{$\begin{array}{l}\mathrm{p} \\
\text { value }\end{array}$} \\
\hline & & + & $\%$ & & \\
\hline Gender & & & & 1.1835 & 0.277 \\
\hline Male & 73 & 39 & 53.4 & & \\
\hline Female & 39 & 25 & 64.1 & & \\
\hline Age (years) & & & & 0.0133 & 0.908 \\
\hline$\geq 60$ & 74 & 42 & 56.8 & & \\
\hline$<60$ & 38 & 22 & 57.9 & & \\
\hline Tumor size $(\mathrm{cm})$ & & & & 0.4585 & 0.498 \\
\hline$\geq 5$ & 47 & 25 & 53.2 & & \\
\hline$<5$ & 62 & 37 & 59.7 & & \\
\hline Insufficient data & 3 & 2 & & & \\
\hline Tumor location & & & & 1.9870 & 0.370 \\
\hline Colon & 57 & 32 & 56.1 & & \\
\hline Rectum & 51 & 31 & 60.8 & & \\
\hline Ileocecal junction & 4 & 1 & & & \\
\hline Histological type & & & & 2.3425 & 0.126 \\
\hline Adenocarcinoma & 102 & 56 & 54.9 & & \\
\hline Non-adenocarcinoma & 10 & 8 & 80.0 & & \\
\hline Tumor differentiation & & & & 3.3634 & 0.186 \\
\hline Well & 1 & 0 & 0.0 & & \\
\hline Moderately & 92 & 55 & 59.8 & & \\
\hline Poorly & 15 & 6 & 40.0 & & \\
\hline Insufficient data & 4 & 3 & & & \\
\hline Serum CEA level (ng/ml) & & & & 0.2855 & 0.593 \\
\hline$\geq 15$ & 14 & 7 & 50.0 & & \\
\hline$<15$ & 71 & 41 & 57.7 & & \\
\hline Insufficient data & 27 & 16 & & & \\
\hline $\mathrm{T}$ (Primary tumor) & & & & 3.0490 & 0.218 \\
\hline $\mathrm{T} 1$ & 7 & 6 & 85.7 & & \\
\hline $\mathrm{T} 2$ & 26 & 16 & 61.5 & & \\
\hline T3 & 79 & 42 & 53.2 & & \\
\hline N (Lymph node metastasis) & & & & 5.2438 & $0.022^{*}$ \\
\hline Positive & 44 & 31 & 70.5 & & \\
\hline Negative & 68 & 33 & 48.5 & & \\
\hline M (Distant metastasis) & & & & 0.6280 & 0.428 \\
\hline Positive & 5 & 2 & 40.0 & & \\
\hline Negative & 107 & 62 & 57.9 & & \\
\hline TNM stage & & & & 9.2855 & $0.026^{*}$ \\
\hline Stage I & 29 & 18 & 62.1 & & \\
\hline Stage II & 36 & 14 & 38.9 & & \\
\hline Stage III & 42 & 30 & 71.4 & & \\
\hline Stage IV & 5 & 2 & 40.0 & & \\
\hline
\end{tabular}

${ }^{*} p<0.05$ 


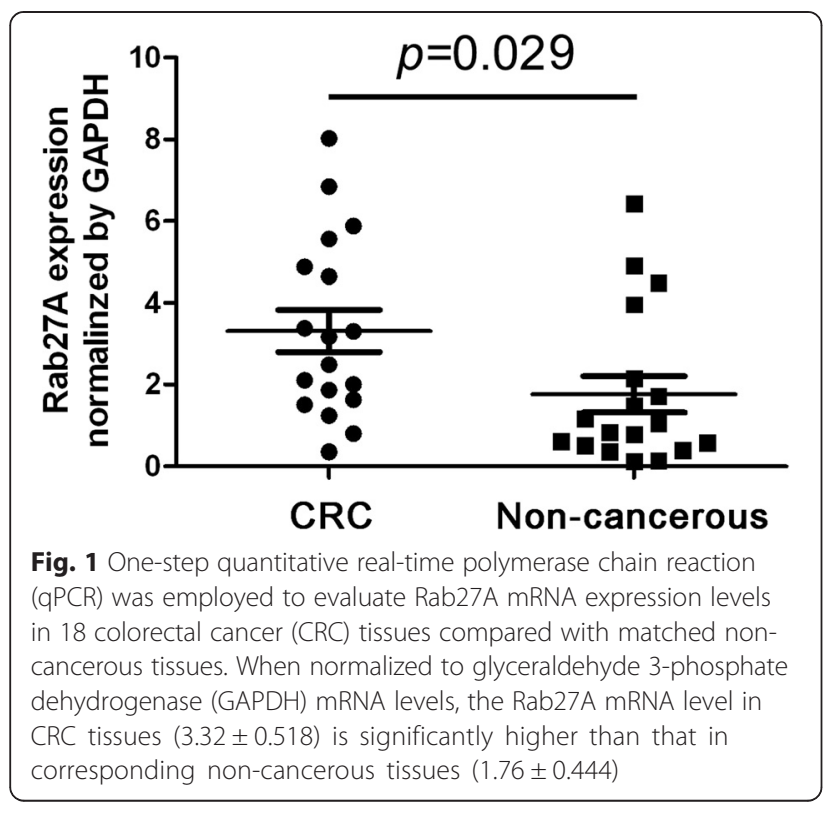

Fig. 1 One-step quantitative real-time polymerase chain reaction (qPCR) was employed to evaluate Rab27A mRNA expression levels in 8 colorectal cancer (CRC) tissues compared with matched noncorresponding non-cancerous tissues $(1.76 \pm 0.444)$ was detected. As is shown in Fig. 2, positive IHC staining was mainly localized in the cytoplasm and nucleus of CRC cells.

\section{Association between Rab27A protein expression and clinicopathological items}

The association between high Rab27A protein expression and representative clinicopathological items was summarized in Table 1. High Rab27A protein expression was statistically related to $\mathrm{N}(p=0.022)$ and TNM stage $(p=0.026)$. Relatively, other clinical items, such as gender, age, tumor size and location, histological type and tumor differentiation, serum CEA level and N, were rarely correlated with high Rab27A protein expression (Table 1).

\section{Survival analysis}

According to univariate analysis, several factors were correlated with overall survival of 112 CRC patients, including Rab27A protein expression $(p=0.001)$, tumor differentiation $(p=0.001)$, serum CEA level $(p=0.006)$, $\mathrm{T}(p=0.004), \mathrm{M}(p=0.005)$ and TNM stage $(p=0.003)$. Moreover, multivariate analysis was executed and the results showed that Rab27A protein expression ( $p=$ $0.012)$ and tumor differentiation $(p=0.004)$ were two

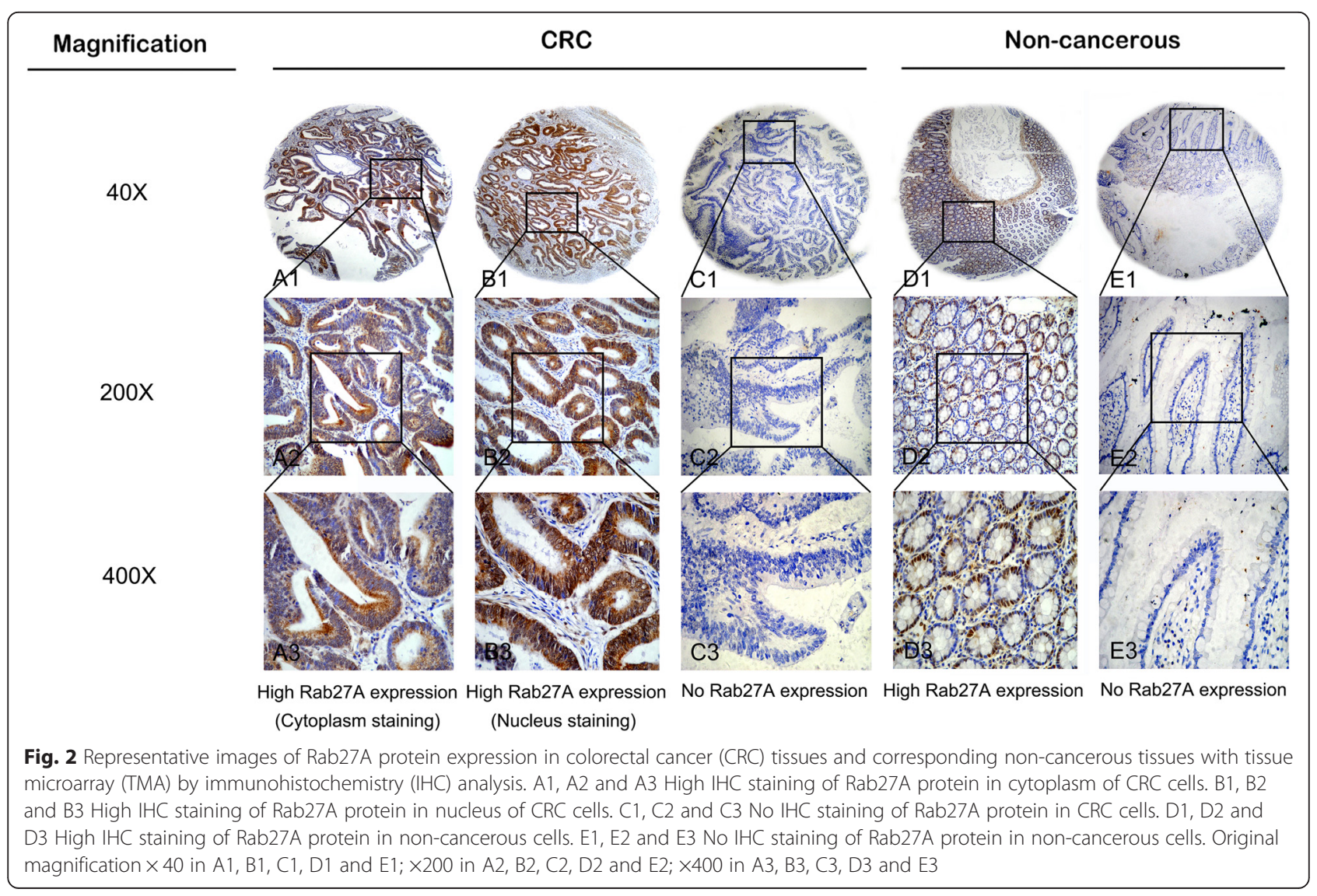


Table 2 Univariate and multivariate analysis of prognostic factors in 112 CRC patients for overall survival

\begin{tabular}{|c|c|c|c|c|c|c|}
\hline & \multicolumn{3}{|c|}{ Univariate analysis } & \multicolumn{3}{|c|}{ Multivariate analysis } \\
\hline & $\mathrm{HR}$ & $p$ value & $95 \% \mathrm{Cl}$ & $H R$ & $p$ value & $95 \% \mathrm{Cl}$ \\
\hline \multicolumn{7}{|l|}{ Rab27A protein expression } \\
\hline High versus Low or no & 0.33 & $0.001^{*}$ & $0.181-0.616$ & 0.36 & $0.012^{*}$ & $0.159-0.794$ \\
\hline \multicolumn{7}{|l|}{ Gender } \\
\hline Male versus Female & 1.43 & 0.277 & $0.750-2.726$ & & & \\
\hline \multicolumn{7}{|l|}{ Age (years) } \\
\hline$\geq 60$ versus $<60$ & 1.16 & 0.642 & $0.618-2.184$ & & & \\
\hline \multicolumn{7}{|l|}{ Tumor size $(\mathrm{cm})$} \\
\hline$\geq 5$ versus $<5$ & 1.21 & 0.537 & $0.659-2.224$ & & & \\
\hline \multicolumn{7}{|l|}{ Tumor location } \\
\hline Colon versus Rectum versus lleocecal junction & 1.49 & 0.131 & $0.888-2.495$ & & & \\
\hline \multicolumn{7}{|l|}{ Histological type } \\
\hline Adenocarcinoma versus Non-adenocarcinoma & 2.40 & 0.227 & $0.581-9.903$ & & & \\
\hline \multicolumn{7}{|l|}{ Tumor differentiation } \\
\hline Well-Moderately versus Poorly & 0.23 & $0.001^{*}$ & $0.117-0.449$ & 0.30 & $0.004^{*}$ & $0.134-0.689$ \\
\hline \multicolumn{7}{|l|}{ Serum CEA level (ng/ml) } \\
\hline$\geq 15$ versus $<15$ & 2.83 & $0.006^{*}$ & $1.356-5.894$ & 1.64 & 0.228 & $0.735-3.639$ \\
\hline \multicolumn{7}{|l|}{ T (Primary tumor) } \\
\hline $\mathrm{T} 1$ versus $\mathrm{T} 2$ versus $\mathrm{T} 3$ & 0.31 & $0.004^{*}$ & $0.140-0.692$ & 0.70 & 0.566 & $0.201-2.406$ \\
\hline \multicolumn{7}{|l|}{ N (Lymph node metastasis) } \\
\hline Positive versus Negative & 1.41 & 0.257 & $0.780-2.533$ & & & \\
\hline \multicolumn{7}{|l|}{ M (Distant metastasis) } \\
\hline Positive versus Negative & 4.35 & $0.005^{*}$ & $1.542-12.171$ & 2.53 & 0.281 & $0.467-13.772$ \\
\hline \multicolumn{7}{|l|}{ TNM stage } \\
\hline Stage I versus Stage II versus Stage III versus Stage IV & 0.58 & $0.003^{*}$ & $0.403-0.829$ & 0.68 & 0.281 & $0.333-1.375$ \\
\hline
\end{tabular}

${ }^{*} p<0.05$
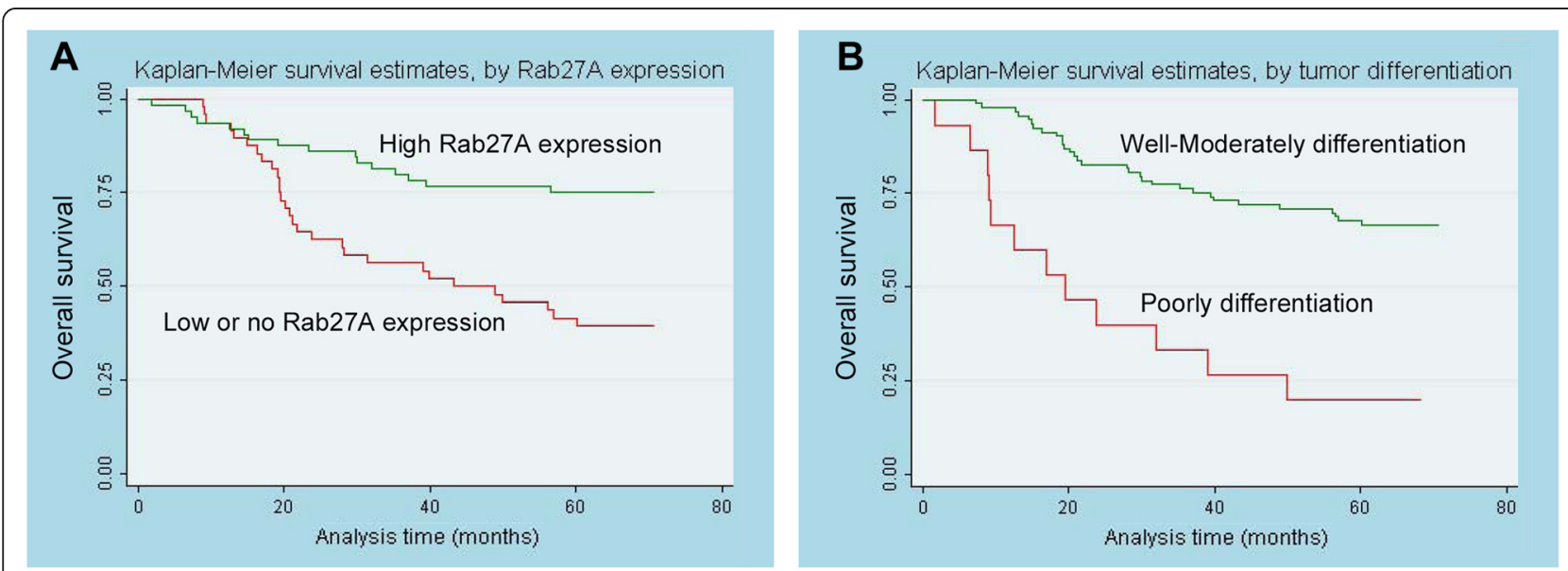

Fig. 3 Survival analysis of 112 colorectal cancer (CRC) patients by Kaplan-Meier method. a Overall survival rate in patients with high Rab27A protein expression (green line) was significantly higher than that in patients with low or no Rab27A expression (red line). b Overall survival rate in patients with well-moderately differentiation of tumor (green line) was significantly higher than that in patients with poorly differentiation of tumor (red line) 
independent predictors of overall survival (Table 2). Kaplan-Meier survival curves in Fig. 3 further demonstrated that CRC patients with high Rab27A protein expression and well-moderately tumor differentiation encountered significantly favorable survival time.

\section{Discussion}

Recently, the roles of several members of the Rab family in human cancer have drawn great attention, including Rab23 and Rab25 [28-30]. As for Rab27A, high expression of Rab27A promoted invasiveness and metastasis of breast cancer cells by secretion of insulin-like growth factor-II [14]. Rab27A modulated the tumor microenvironment and facilitated tumor development by regulating exocytosis of multivesicular endosomes and lead to exosome secretion [17]. For glioma, Rab27A acted as an oncogenic factor and significantly associated with tumor progression and poor prognosis in all grades of gliomas [18]. Similarly, in HCC, Rab27A expression was closely correlated with HCC progression and can be used as a valuable prognostic indicator for HCC patients [19]. Hence it is rationale to presume that Rab27A also performs in CRC and there are certain relationships between Rab27A expression and certain clinical features of CRC patients.

The results of qPCR test with small samples of CRC showed that Rab27A expression in CRC samples was statistically increased than that in non-cancerous tissues. In the subsequent IHC analysis, the results were consistent with the previous qPCR test and the protein expression of Rab27A in CRC TMA was also higher than that in non-cancerous tissues. The data of differential expression of Rab27A in CRC in this present study was consistent with the content of previous reports which stated that expression of Rab27A was significant higher in glioma and HCC $[18,19]$. In addition, high Rab27A protein expression do correlated with certain substantial clinical attributes, including $\mathrm{N}$ and TNM stage. The above data agreed with previous researches and supported the promotive function of Rab27A in tumorigenesis $[16,18,19]$.

In the survival analyses of our present study, univariate analysis revealed that several substantial items correlated with overall survival of 112 CRC patients. Multivariate analysis further elucidated that Rab27A protein expression and tumor differentiation may identified as two independent prognostic factors for CRC prognosis in this present study. Thoroughly, Kaplan-Meier curve indicated that CRC patients with high Rab27A expression had a significantly better prognosis than that of patients with low or no expression. The data of survival analysis were in line with the previous study, which mentioned the prognostic effect of Rab27A in GC and CRC (without providing the exact survival data) [19].
So far, there are still some inconsistencies even contradictories concerning the effects of Rab27A in human cancers. Hendrix et al. reported no expression of Rab27A was detected in breast cancer [31] while Dong et al. reported that Rab27A expression was higher in primary HCC than in matched adjacent tissue [19]. High expression of Rab27A showed poor survival in gliomas and HCC as well as suggested favorable survival in GC and CRC simultaneously $[18,19]$. In my view, these discrepant findings indicate that Rab27A may have different molecular mechanisms in different human cancer under specific circumstances. For instance, the secretion of exosome critically needs the present of Rab27A and the functions of tumor exosomes were dual. For one thing, exosomes could transfer tumor antigens to dendritic cells for presentation of these antigens to $\mathrm{T}$ lymphocytes [32]. For another, exosomes showed inhibitory functions on effector immune responses as well as promoted metastasis [33, 34]. The multiple activities of exosome may explain the complicated function of Rab27A in human cancer. Further researches that enroll larger cancer samples and elucidate the potential mechanisms of Rab27A performance are of great importance to prove our presumption.

In summary, to our best knowledge, this study was the first to report on the differential expression of Rab27A in CRC and our data indicated that Rab27A may be identified as a prognostic biomarker in CRC patients.

\section{Conclusions}

In conclusion, this is the first report of the differential expression of Rab27A in CRC. High Rab27A expression was detected in CRC tissues, and that CRC patients with elevated Rab27A expression were prone to suffer malignant behaviors including positive $\mathrm{N}$ and advanced TNM stage. Moreover, high expression of Rab27A suggested favorable prognosis in CRC patients.

\section{Competing interests \\ The authors declare that they have no competing interests.}

\section{Authors' contributions}

PSC conceived and designed the study; CBS, XJY, NH, LX (Lin Xiong) and HJZ performed the experiments; YJN, LX (Li XU), FZ and HJZ performed patient collection and clinical data interpretation; CBS, XJY, YJN and FZ performed the statistical analysis; CBS drafted the paper; PSC supervised the study. CBS and XJY contributed equally to this study and they are co-first authors. All authors read and approved the final paper.

\section{Author details}

'Department of Pathology and Pathophysiology, School of Medicine, Southeast University, Nanjing 210009, China. ${ }^{2}$ Department of General Surgery, the Second Affiliated Hospital of Nanjing Medical University, Nanjing 210011, China. ${ }^{3}$ Department of Traumatic Surgery, Changzhou No. 2 People's Hospital Affiliated with Nanjing Medical University, Changzhou 213000, China. ${ }^{4}$ Department of Pathology, Jiangsu Cancer Hospital, Nanjing 210000, China. ${ }^{5}$ Department of Hepatobiliary and Laparoscopic Surgery, YiXing People's Hospital, the Affiliated YiXing Hospital of Jiangsu University, Yixing 214200, China. ${ }^{6}$ Department of Pathology and Laboratory Medicine, the 
Affiliated Hospital of Nantong University, Nantong 226000, China.

${ }^{7}$ Department of Pathology, the Second Affiliated Hospital of Nanjing Medical University, Nanjing 210011, China. ${ }^{8}$ The Key Laboratory of Cancer Biomarkers, Prevention \& Treatment Cancer Center and The Key Laboratory of Antibody Technique of Ministry of Health, Nanjing Medical University, Nanjing 210029, China.

Received: 28 October 2014 Accepted: 29 May 2015

Published online: 13 June 2015

\section{References}

1. Jemal A, Bray F, Center MM, Ferlay J, Ward E, Forman D. Global cancer statistics. CA: a cancer journal for clinicians. 2011;61(2):69-90.

2. Huang CW, Tsai HL, Chen YT, Huang CM, Ma CJ, Lu CY, et al. The prognostic values of EGFR expression and KRAS mutation in patients with synchronous or metachronous metastatic colorectal cancer. BMC cancer. 2013;13:599.

3. Fang YJ, Wu XJ, Zhao Q, Li LR, Lu ZH, Ding PR, et al. Hospital-based colorectal cancer survival trend of different tumor locations from 1960s to 2000s. PloS one. 2013;8(9), e73528.

4. Kleespies A, Fuessl KE, Seeliger $H$, Eichhorn ME, Muller MH, Rentsch M, et al. Determinants of morbidity and survival after elective non-curative resection of stage IV colon and rectal cancer. Int J Colorectal Dis. 2009;24(9):1097-109.

5. Platell C, Ng S, O'Bichere A, Tebbutt N. Changing management and survival in patients with stage IV colorectal cancer. Diseases of the colon and rectum. 2011;54(2):214-9.

6. Karoui M, Roudot-Thoraval F, Mesli F, Mitry E, Aparicio T, Des Guetz G, et al. Primary colectomy in patients with stage IV colon cancer and unresectable distant metastases improves overall survival: results of a multicentric study. Diseases of the colon and rectum. 2011;54(8):930-8.

7. Atkin WS, Edwards R, Kralj-Hans I, Wooldrage K, Hart AR, Northover JM, et al. Once-only flexible sigmoidoscopy screening in prevention of colorectal cancer: a multicentre randomised controlled trial. Lancet. 2010;375(9726):1624-33.

8. Chen C, Wang L, Liao Q, Huang Y, Ye H, Chen F, et al. Hypermethylation of EDNRB promoter contributes to the risk of colorectal cancer. Diagnostic pathology. 2013;8:199.

9. Li Y, Wei J, Xu C, Zhao Z, You T. Prognostic significance of cyclin d1 expression in colorectal cancer: a meta-analysis of observational studies. PloS one. 2014;9(4), e94508.

10. Stenmark H. Rab GTPases as coordinators of vesicle traffic. Nature reviews Molecular cell biology. 2009;10(8):513-25.

11. Hutagalung AH, Novick PJ. Role of Rab GTPases in membrane traffic and cell physiology. Physiological reviews. 2011;91(1):119-49.

12. Fukuda M. Rab27 effectors, pleiotropic regulators in secretory pathways. Traffic. 2013;14(9):949-63.

13. Van Gele M, Dynoodt P, Lambert J. Griscelli syndrome: a model system to study vesicular trafficking. Pigment cell \& melanoma research. 2009;22(3):268-82.

14. Wang JS, Wang FB, Zhang QG, Shen ZZ, Shao ZM. Enhanced expression of Rab27A gene by breast cancer cells promoting invasiveness and the metastasis potential by secretion of insulin-like growth factor-II. Molecular cancer research : MCR. 2008;6(3):372-82.

15. Montel V, Huang TY, Mose E, Pestonjamasp K, Tarin D. Expression profiling of primary tumors and matched lymphatic and lung metastases in a xenogeneic breast cancer model. The American journal of pathology. 2005;166(5):1565-79.

16. Wu X, Hu A, Zhang M, Chen Z. Effects of Rab27a on proliferation, invasion, and anti-apoptosis in human glioma cell. Tumour biology : the journal of the International Society for Oncodevelopmental Biology and Medicine. 2013;34(4):2195-203.

17. Bobrie A, Krumeich S, Reyal F, Recchi C, Moita LF, Seabra MC, et al. Rab27a supports exosome-dependent and -independent mechanisms that modify the tumor microenvironment and can promote tumor progression. Cancer research. 2012;72(19):4920-30.

18. Wang H, Zhao Y, Zhang C, Li M, Jiang C, Li Y. Rab27a was identified as a prognostic biomaker by mRNA profiling, correlated with malignant progression and subtype preference in gliomas. PloS one. 2014;9(2), e89782.

19. Dong WW, Mou Q, Chen J, Cui JT, Li WM, Xiao WH. Differential expression of Rab27A/B correlates with clinical outcome in hepatocellular carcinoma. World journal of gastroenterology : WJG. 2012;18(15):1806-13.
20. Luo B, Yun X, Fan R, Lin YD, He SJ, Zhang QM, et al. Cancer testis antigen OY-TES-1 expression and serum immunogenicity in colorectal cancer: its relationship to clinicopathological parameters. International journal of clinical and experimental pathology. 2013;6(12):2835-45.

21. Fu M, Fan W, Pu X, Ni H, Zhang W, Chang F, et al. Elevated expression of SHIP2 correlates with poor prognosis in non-small cell lung cancer. International journal of clinical and experimental pathology. 2013;6(10):2185-91.

22. Fu M, Gu X, Ni H, Zhang W, Chang F, Gong L, et al. High expression of inositol polyphosphate phosphatase-like 1 associates with unfavorable survival in hepatocellular carcinoma. International journal of clinical and experimental pathology. 2013;6(11):2515-22

23. Mao Y, Zhang DW, Zhu H, Lin H, Xiong L, Cao Q, et al. LMP1 and LMP2A are potential prognostic markers of extranodal NK/T-cell lymphoma, nasal type (ENKTL). Diagnostic pathology. 2012;7:178

24. Zhang H, Qiu J, Ye C, Yang D, Gao L, Su Y, et al. ROR1 expression correlated with poor clinical outcome in human ovarian cancer. Scientific reports. 2014;4:5811.

25. Lin H, Zhang H, Wang J, Lu M, Zheng F, Wang C, et al. A novel human Fab antibody for Trop2 inhibits breast cancer growth in vitro and in vivo. International journal of cancer Journal international du cancer. 2014;134(5):1239-49.

26. Zhu H, Lu J, Wang $X$, Zhang $H$, Tang $X$, Zhu J, et al. Upregulated ZO-1 correlates with favorable survival of gastrointestinal stromal tumor. Medical oncology. 2013;30(3):631.

27. Gu X, Fu M, Ge Z, Zhan F, Ding Y, Ni H, et al. High expression of MAGE-A9 correlates with unfavorable survival in hepatocellular carcinoma. Scientific reports. 2014:4:6625.

28. Cheng KW, Lahad JP, Kuo WL, Lapuk A, Yamada K, Auersperg N, et al. The RAB25 small GTPase determines aggressiveness of ovarian and breast cancers. Nature medicine. 2004;10(11):1251-6.

29. Fan $Y, X$ in $X Y$, Chen BL, Ma X. Knockdown of RAB25 expression by RNAi inhibits growth of human epithelial ovarian cancer cells in vitro and in vivo. Pathology. 2006;38(6):561-7.

30. Hou Q, Wu YH, Grabsch H, Zhu Y, Leong SH, Ganesan K, et al. Integrative genomics identifies RAB23 as an invasion mediator gene in diffuse-type gastric cancer. Cancer research. 2008;68(12):4623-30.

31. Hendrix A, Maynard D, Pauwels P, Braems G, Denys H, Van den Broecke R, et al. Effect of the secretory small GTPase Rab27B on breast cancer growth, invasion, and metastasis. Journal of the National Cancer Institute. 2010;102(12):866-80.

32. Zeelenberg IS, Ostrowski M, Krumeich S, Bobrie A, Jancic C, Boissonnas A, et al. Targeting tumor antigens to secreted membrane vesicles in vivo induces efficient antitumor immune responses. Cancer research. 2008;68(4):1228-35.

33. Taylor DD, Gercel-Taylor C. Exosomes/microvesicles: mediators of cancer-associated immunosuppressive microenvironments. Seminars in immunopathology. 2011;33(5):441-54.

34. Peinado H, Aleckovic M, Lavotshkin S, Matei I, Costa-Silva B, MorenoBueno $G$, et al. Melanoma exosomes educate bone marrow progenitor cells toward a pro-metastatic phenotype through MET. Nature medicine. 2012;18(6):883-91.

\section{Submit your next manuscript to BioMed Central and take full advantage of:}

- Convenient online submission

- Thorough peer review

- No space constraints or color figure charges

- Immediate publication on acceptance

- Inclusion in PubMed, CAS, Scopus and Google Scholar

- Research which is freely available for redistribution 